\title{
Breast Solid Neuroendocrine Carcinoma
}

National Cancer Institute

\section{Source}

National Cancer Institute. Breast Solid Neuroendocrine Carcinoma. NCI Thesaurus. Code C97965.

A neuroendocrine carcinoma that arises from the breast. It is characterized by the presence of cellular solid nests and trabeculae that contain spindle-shaped, plasmacytoid, and large neuroendocrine cells. 